\title{
Dynamics of Morphological Changes in the Anterior Segment of the Rat Eye after Experimental Alkali Burn Depending on the Acetylator Phenotype
}

\author{
Khalidjan M. Kamilov, PhD,ScD; Laylo M. Maksudova* \\ Tashkent Institute of Postgraduate Medical Education \\ Tashkent, Uzbekistan
}

\begin{abstract}
The purpose of this study was to identify the nature and dynamics of morphological changes in the anterior segment of the rat eye (ASE) in the aspect of regeneration opportunities under an experimental alkali burn (EAB) in the fast and slow acetylators.

Materials and Methods: The experiment was performed on 50 adult outbred male Wistar rats weighting from 180 to $200 \mathrm{~g}$. According to results of testing, all animals were divided into 2 groups: fast acetylators and slow acetylators. The experimental chemical eye burn model was performed according to standard procedures by applying a $10 \% \mathrm{NaOH}$ solution for 10 seconds. The tissues of ASE were the object of this study. The morphological examination was performed on the 3rd, 5th, 12th, and 30th day after EAB.

Results: The morphological changes of eye tissues after EAB are formed immediately after the injury involving the deep tissues and are characterized by severe manifestations of the colliquative burn of all eye structures. The intensity of the pathological process increases over time with subsequent reduction of pathological changes on day 30 post-injury. The intensity of morphological changes and their dynamics vary depending on the N-acetylation, given equal external conditions. Regarding the features of drug biotransformation in slow acetylators, it is advisable to take acetylation phenotype into account when choosing the treatment method. (Int J Biomed. 2015;5(4):219-223.)
\end{abstract}

Keywords: rat's eye; alkali burn; morphological changes; acetylation phenotype.

\section{Introduction}

Injuries and burns of the cornea over the past three decades have been the leading cause of eye injuries $(68 \%$ to $70 \%)$ [1-4]. The structural and functional restoration of a chemical burn-injured cornea remains challenging. In severe cases, the eye limbus and central epithelium can be lost, leading to loss of vision. Slow epithelialization, persistent ulceration, corneal perforation and angiogenesis are the main complications and result from the processes of inflammation, neovascularization, and conjunctivalization of cornea $[1,2,5]$. Strategies to treat corneal chemical burns include antibiotics, tear substitutes, corticosteroids, ascorbic acid, collagenase inhibitors and surgical treatments such as penetrating keratoplasty and amniotic membrane transplantation [6].

*Corresponding author: Laylo M. Maksudova. Tashkent Institute of Postgraduate Medical Education. Tashkent, Uzbekistan. E-mail:doclaylo@rambler.ru
However, the structural and functional restoration of alkali burn-injuries to the cornea remains a challenge, despite these therapies and thus prompting the search for novel treatment strategies [7-9]. A detailed study of regenerative processes under the influence of various drugs is a top priority of modern ophthalmology.

Our analysis of the contemporary literature on the study of morphological changes in the organ of vision under the influence of chemical burns has identified some common features in the views of researchers on this problem. In burn injuries, the focus of interest of the majority of contemporary researchers is the final result of the application of a pharmacological agent without analysis of the dynamics of the processes. In most cases, the final result is expressed as: (a) negative result - no effect of the pharmacological treatment; (b) moderate result - an insufficient (incomplete) effect of the pharmacological treatment; (c) positive result - a complete recovery of visual functions during pharmacological treatment. It should be noted that for the patient only complete 
recovery of vision might be considered an effective treatment, while moderate or insufficient effect is a doctor's defeat in the fight for the preservation of vision.

The effect of treatment depends on the drug metabolism and rate of elimination of toxic products of chemical burns. Many pharmacological agents, as well as some toxic products of chemical burns, are metabolized primarily by acetylation by liver $\mathrm{N}$-acetyltransferase. The rate of acetylation is genetically determined. The quantitative assessment of the rate and extent of acetylation of many chemical substances yields bimodal distribution, representing fast and slow acetylators [10-12]. Recently, it has been suggested that slow acetylators are at greater risk of developing severe pathological processes $[13,14]$. But there have been no studies of the association between the dynamics of morphological changes during reparative processes with alkali burns of the eyes, on the one hand, and genetic differences in xenobiotic elimination and metabolic rate on the other.

Based on the above, the purpose of this study was to identify the nature and dynamics of morphological changes in the anterior segment of the rat eye (ASE) in the aspect of regeneration opportunities under an experimental alkali burn $(\mathrm{EAB})$ in the fast and slow acetylators.

\section{Materials and Methods}

The study was conducted between October 2013 and May 2014 in accordance with the principles of ARVO Statement for the use of animals in ophthalmic and visual research. The experiment was performed on 50 adult outbred male Wistar rats weighting from 180 to $200 \mathrm{~g}$. Acetylation phenotype (AF) was determined according to the Brodie and Axelrod method (1949) as modified by T.A. Popova and O.B. Leonenko (1977), using Sulfathiazole, by analyzing the drug metabolite ratio in urine after per os administration of $10 \mathrm{mg} / \mathrm{kg}$ Sulfathiazole. According to results of testing, all animals were divided into 2 groups: fast acetylators (FAs) and slow acetylators (SAs). The study group consisted of 20 slow acetylator rats (SARs) and 20 fast acetylator rats (FARs) (Fig.1-2).

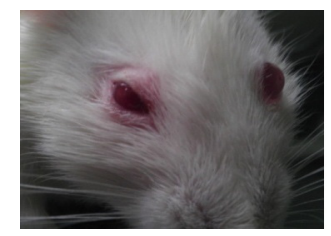

Fig.1. The eye of SAR. EAB.

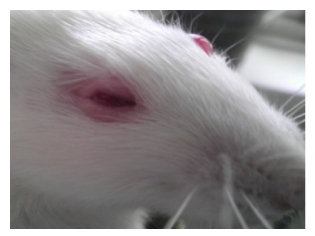

Fig. 2. The eye of FAR. EAB
In the study group, the experimental chemical eye burn model was performed according to standard procedures by applying a $10 \% \mathrm{NaOH}$ solution for 10 seconds. The control group consisted of $5 \mathrm{SARs}$ and 5 FARs who underwent application of a $0.9 \% \mathrm{NaCl}$ solution in the same exposure. All animals were kept in the same vivarium conditions. The tissues of ASE were the object of this study. The morphological examination was performed on the 3rd, 5th, 12th, and 30th day after EAB. Procedures for euthanasia were performed according to MMRI Policy for the Humane Care and Use of Laboratory Animals in a manner consistent with

the recommendations of the American Veterinary Medical Association (AVMA) Panel on Euthanasia, unless a deviation is justified for scientific reasons in writing by the investigator".

Morphological studies were conducted using standard light microscopy techniques (H\&E staining). Light optical micrographs obtained with different magnifications using microscopes Carl Zeiss AxioScope, Biolam-I, and Biolam I2 combined with a digital camera.

\section{Results}

\section{Dynamics of morphological changes in ASE of FARs after EAB}

Figure 3 presents the morphological picture of the healthy rat cornea. On the third day after EAB, morphological changes in the cornea of FARs were characterized by the development of edema with the separation and dissociation of collagen fibers in the central area of burn injury (Fig.4). Cellular elements of the anterior corneal epithelium were completely absent. The lamina limitans anterior, having a fibrillar structure, was partially preserved. The corneal stroma was represented by irregularly aligned connective tissue plates, which intersected at different angles and consisted of the separated and dissociated collagen fibers of different thicknesses. The posterior corneal epithelium, consisting of flat polygonal cells, was also edematous and amorphous in most cases and separated from the Descemet's membrane. Revealed morphological changes allow us to conclude that rude and often irreversible structural changes in the affected tissues of the eye are formed by the second day after EAB.

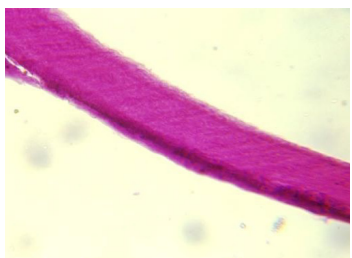

Fig. 3. The morphological picture of the healthy rat cornea. H\&E, $10 \times 10$.

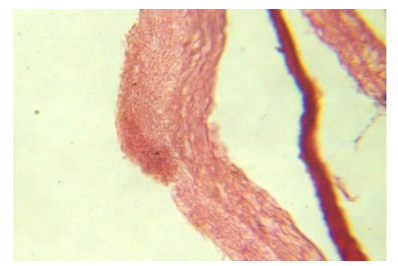

Fig. 4. Exfoliation of the anterior corneal epithelium. FA. The $3^{\text {rd }}$ day after EAB. H\&E.10x40.

On the fifth day after EAB, the infiltration and ulceration of the burn surface of the cornea were intensified, and signs of edema with the involvement of the large areas of the corneal stroma increased. The anterior corneal epithelium was absent in the central zone of edema, and, toward the periphery, the transition from a single-layer epithelium into the two- or pseudostratified epithelium was observed. Areas of degradation in the corneal stroma were identified (Fig. 5 and 6).

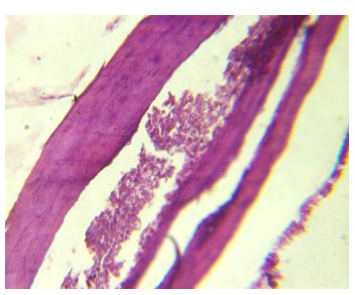

Fig.5. Edema and areas of degradation in the corneal stroma. The 5th day after EAB. FA. H\&E. $10 \times 16$.

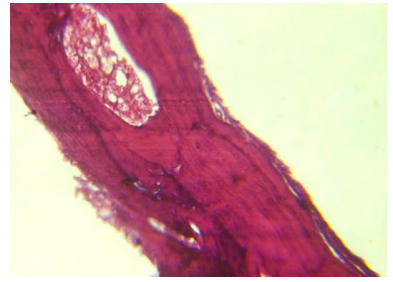

Fig.6. Elements of the corneal stroma destruction, the surface erosions. The 5th day after EAB. FA. H\&E. $10 \times 16$. 
It should be noted that hyperemia and overflow of blood capillaries, causing edema severity, are characteristic morphological changes in the eye tissues on the fifth day after EAB.

By the 12th day after EAB, pathological changes in the connective tissue of the sclera had increased and the dissociation with fragmentation of collagen fibers, as well as colliquation of tissues ("colliquatio"), were detected (Fig. 7 and 8).

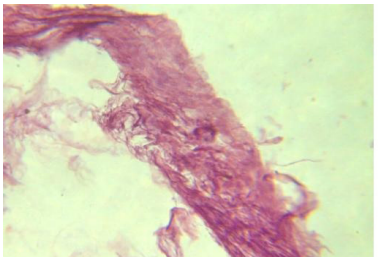

Fig.7. Dissociation with fragmentation in the connective tissue of the sclera. The 12th day after EAB. FA. H\&E.10x16.

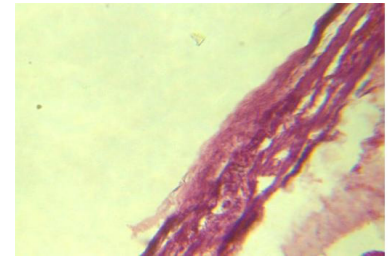

Fig. 8. Signs of scleral cell death. The 12th day after EAB. FA.H\&E. $10 \times 16$.
The absence of morphological signs of scab was manifested by hydrolysis of proteins and cell membranes. Signs of cell death caused by the spread and deep alkali penetration were also identified. All structures of the eye, including the conjunctiva (Fig.9), eyelids (Fig.10), cornea, sclera, lens, and even the retina, were involved in the pathological process. Edema and the leukocyte infiltration of the connective tissue of the eyelids were spread.

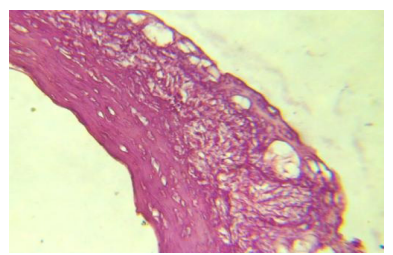
and hyperemia. The 12th day after EAB. FA.H\&E. $10 x 16$.
Fig.9. the conjunctiva: edema

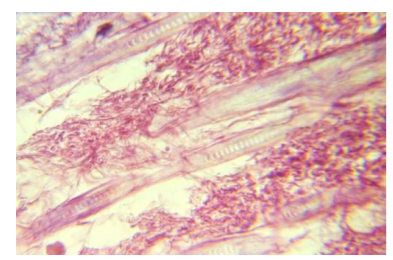

Fig.10. Edema and leukocyte infiltration of the connective tissue of the eyelid. The 12th day after $E A B$. FA. $H \& E .10 \times 16$.

By the 30th day after EAB, tissue swelling persisted, but it was not so pronounced. The phenomena of tissue destruction came to the fore, namely the destruction of the accommodative apparatus with hemorrhagic manifestations of varying severity from an imbibition to the extensive hemorrhages (Fig. 11-12).

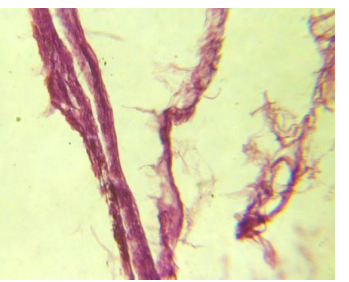

Fig. 11. Dystrophy of the elastic fibers; the bundle and necrosis of the sclera. The 30th day after EAB. FA. H\&E. $10 \times 16$

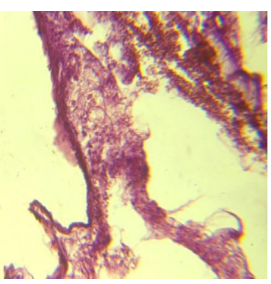

Fig. 12. The anterior chamber angle. Edema and destruction of accommodative apparatus. The 30th day after EAB. FA. 10x16

\section{Dynamics of morphological changes in ASE of SARs after EAB}

On the third day after EAB, the morphological changes in the rat cornea were characterized by the formation of

pronounced edema. The multiple areas of small erosions on the outer surface of the cornea with elements of cellular debris were detected (Fig.13). Sclera was characterized by edema; collagen fibers were loose and had relatively parallel positions to the surface of the eyeball (Fig.14). The outer surface of the sclera was eroded in some places, so that the free edges of the fibrous tissue were behind the epithelium anterior which had a tendency to separate from the stroma. The auxiliary apparatus of the eye, eyelids, and lacrimal apparatus were also involved in the pathological process (Fig.15). On the fifth day after $\mathrm{EAB}$, symptoms of edema were intensified; there were tears and a lamination of the corneal fibers; an accumulation of large amounts of blood cells, mainly red blood cells, was identified in the lumen of the tears (Fig.16).

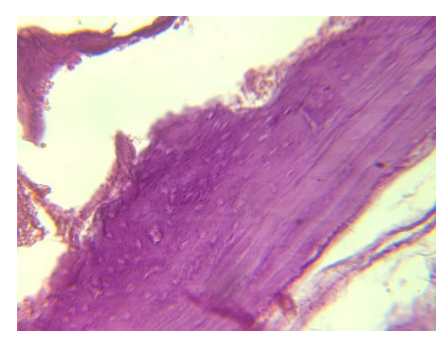

Fig.13. The cornea. The 3rd day after $E A B$. SA. H\&E. $10 \times 16$

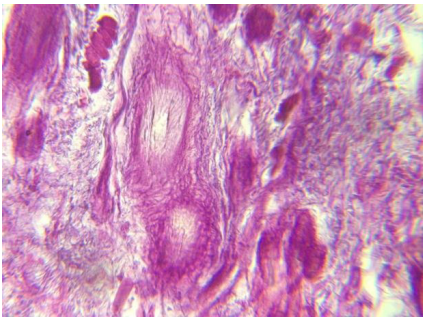

Fig.15. Eyelid, hair. Stasis and sludge of erythrocytes. The $3 r d$ day after EAB. SA. 10x16

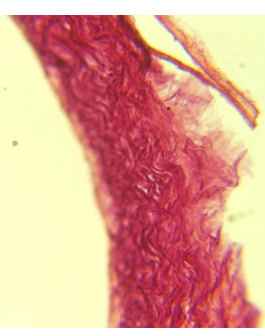

Fig.14. The sclera. The 3rd day after EAB. SA. H\&E. $10 x 16$

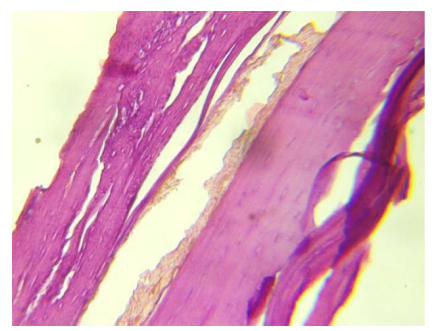

Fig.16. Edema tears and a lamination of the corneal fibers. The 5st day after EAB. SA. 10x16.

Morphological changes in the angle of the eye were characterized by a diffuse increase of edema. The cavities of venous sinuses of sclera and Schlemm's canal were empty; blood cells were absent in the wide vascular lumens (Fig.17). The spread of the alkali burn went deep into the tissues of the eye with involvement in the pathological process of the entire organ, damaging the receptor and neurosensory apparatus. We have identified the characteristic changes in the form of increasing diffuse edema in the tissues that were infiltrated with blood (imbibitions with red blood cells); areas of hemorrhage were also found. Blood cells were found within the lumen of blood vessels, mainly in parietal areas. Figure 18 presents the stagnation and stasis of blood in the back wall of the eye.

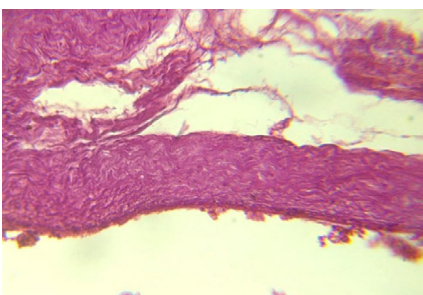

Fig.17. Schlemm's canal. The 5st day after EAB. SA. $10 \times 16$.

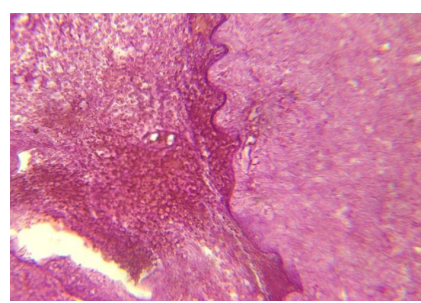

Fig.18. The back wall of the eye. The 5st day after EAB. SA. $10 x 16$. 
On the 12th day after EAB, we identified a progression of degradation processes, signs of epithelial tissue regeneration with the participation of the cellular elements of the nonkeratinized stratified squamous epithelium, the anterior corneal epithelium, that could be traced ubiquitously (Fig.19).

On the 30th day after EAB, we found the negative dynamics characterized by the severe processes of hypotrophy and atrophy, with loss of vision. The morphological picture of trophic tissue disorders was due to processes of the generalized blood circulation disorders with thrombosis on the background of an edema, as well as disturbances of the drainage function of the venous and lymphatic system (Fig.20).
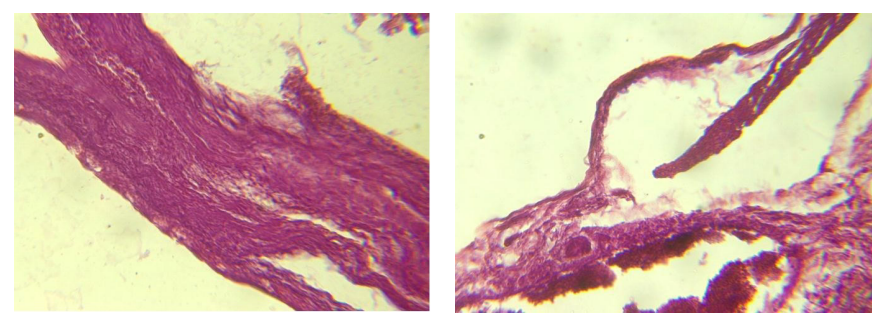

Fig.19. Signs of epithelial tissue Fig.20. Corner of the eye. The 30th regeneration. The 12th day after dayafter EAB. SA. 10x16. EAB. SA. $10 \times 16$.

\section{Discussion}

To analyze of the morphological changes in the tissues of the eye after EAB, it is advisable to remember the fundamentals of the damaging effects of alkalis. Alkalis, leading to saponification of proteins, fats and fatlike substances of cell membranes, penetrate intracellularly and change the plasmalemma cellular $\mathrm{pH}$, which leads to dissolution and colliquation of proteins and determines the degree of degradation. The character of morphological changes in tissues of the eye on the background of EAB is also associated with features of supply and regeneration of the cornea, which occurs due to the diffusion of micronutrients from the front chamber of the eye and blood vessels of the limbus, because of the absence of blood vessels in the cornea. Moreover, against the background of pronounced edema and loss of structure in all histological layers of the cornea, a corneal lymphatic system consisting of narrow gaps usually is isolated from the possibility of adequate communication with the venous plexus.

Our results confirm the previously published data that alkali burns, dissolving tissue proteins and inducing a colliquative necrosis [15-17], quickly penetrate deeply into tissues and the ocular cavity and affect the inner shells of the cavity. According to the authors, some alkalis are found in the anterior chamber of the eye after 5 to 6 minutes following their introduction into the conjunctival cavity. In this regard, we can assume that the possibility of achieving the minimization of dioptric apparatus defeat in the early stages of treatment in FAs does not exclude the penetration of alkali in the deeper layers of the eye. In this case, not only are the cornea and conjunctiva affected, but also the inner shells of the front chamber of the eye. Hemorrhagic manifestations against the backdrop of the ongoing inflammatory response will contribute to the widening of the connective tissue structures and, consequently, a lesion of the functional activity of the accommodative apparatus of the eye, even on the background of the saved morphological organization of the dioptric apparatus.

The differences in the morphological changes in tissues of the eye with alkali burn injuries in animals with different $\mathrm{AF}$, in particular, the severity of the lesions in SARs, may be important to optimize the treatment of this severe injury. Regarding the features of drug biotransformation in SAs, it is advisable to take $\mathrm{AF}$ into account when choosing the treatment method. In particular, we recommend including AF definition in the standards of diagnosis and treatment of chemical burns of the eye in order to predict the possible outcome of therapy and its optimization.

In conclusion, it should be noted that the morphological changes of eye tissues after EAB are formed immediately after the injury involving the deep tissues and are characterized by severe manifestations of the colliquative burn of all eye structures. The intensity of the pathological process increases over time with subsequent reduction of pathological changes on day 30 post-injury. The intensity of morphological changes and their dynamics vary depending on the $\mathrm{N}$-acetylation, given equal external conditions. Regarding the features of drug biotransformation in slow acetylators, it is advisable to take acetylation phenotype into account when choosing the treatment method.

\section{Competing interests}

The authors declare that they have no competing interests.

\section{References}

1. Wagoner MD. Chemical injuries of the eye: current concepts in pathophysiology and therapy. Surv Ophthalmol. 1997; 41(4):275-313.

2. Brodovsky SC, McCarty CA, Snibson G, Loughnan M, Sullivan L, Daniell M, et al. Management of alkali burns: an 11-year retrospective review. Ophthalmology. 2000; 107(10):1829-35.

3. Ramakrishnan KM, Mathivanan T, Jayaraman V, Babu M, Shankar J. Current scenario in chemical burns in a developing country: Chennai, India. Ann Burns Fire Disasters. 2012; 25(1):8-12.

4. Iakymenko S. Forty-five years of keratoprosthesis study and application at the Filatov Institute: a retrospective analysis of 1060 cases. Int J Ophthalmol. 2013; 6(3):375-80.

5. Adamis AP, Aiello LP, D'Amato RA. Angiogenesis and ophthalmic disease. Angiogenesis. 1999; 3(1):9-14.

6. He J, Bazan NG, Bazan HE. Alkali-induced corneal stromal melting prevention by a novel platelet-activating factor receptor antagonist. Arch Ophthalmol. 2006; 124(1):70-8. 7. Han Y, Shao Y, Liu TT, Li SM, Li W, Liu ZG. Therapeutic effects of topical netrin- 4 in a corneal acute inflammatory model. Int J Ophthalmol. 2015; 8(2):228-33.

8. Ke Y, Wu Y, Cui X, Liu X, Yu M, Yang C, Li X, et al. Polysaccharide hydrogel combined with mesenchymal stem cells promotes the healing of corneal alkali burn in rats. PLoS One. 2015; 10(3):e0119725. doi: 10.1371/journal. pone.0119725. eCollection 2015.

9. Yao L, Li ZR, Su WR, Li YP, Lin ML, Zhang WX, Liu Y, 
Wan Q, Liang D. Role of mesenchymal stem cells on cornea wound healing induced by acute alkali burn. PLoS One. 2012; 7(2):e30842. Epub 2012 Feb 17.

10. Evans DA, White TA. Human acetylation polymorphism. J Lab Clin Med. 1964; 63:394-403.

11. Evans DA. An improved and simplified method of detecting the acetylator phenotype. J Med Genet. 1969; 6(4):405-7.

12. Gelber R, Peters JH, Gordon GR, Glazko AJ, Levy L. The polymorphic acetylation of dapsone in man. Clin Pharmacol Ther. 1971; 12(2):225-38.
13. Thomas L. Lemke, David A. Williams. Foye's Principles of Medicinal Chemistry. 7th ed. LWW; 2012

14. NAT2 slow acetylation and bladder cancer risk: a metaanalysis of 22 case-control studies conducted in the general population. Pharmacogenetics. 2000; 10(2):115-22.

15. Sykes RA, Mani MM, Hiebert JM. Chemical burns: retrospective review. J Burn Care Rehabil. 1986; 7(4):343-7. 16. Mozingo DW, Smith AA, McManus WF, Pruitt BA Jr, Mason AD Jr. Chemical burns. J Trauma. 1988; 28(5):642-7. 17. Palao R, Monge I, Ruiz M, Barret JP. Chemical burns: pathophysiology and treatment. Burns. 2010; 36(3):295-304. 